\title{
Uncountable sets and an infinite real number game
}

\author{
Matthew H. Baker ${ }^{1}$ \\ School of Mathematics \\ Georgia Institute of Technology \\ Atlanta, GA 30332-0160 \\ mbaker@math.gatech.edu
}

The game. Alice and Bob decide to play the following infinite game on the real number line. A subset $S$ of the unit interval $[0,1]$ is fixed, and then Alice and Bob alternate playing real numbers. Alice moves first, choosing any real number $a_{1}$ strictly between 0 and 1 . Bob then chooses any real number $b_{1}$ strictly between $a_{1}$ and 1 . On each subsequent turn, the players must choose a point strictly between the previous two choices. Equivalently, if we let $a_{0}=0$ and $b_{0}=1$, then in round $n \geq 1$, Alice chooses a real number $a_{n}$ with $a_{n-1}<a_{n}<b_{n-1}$, and then Bob chooses a real number $b_{n}$ with $a_{n}<b_{n}<b_{n-1}$. Since a monotonically increasing sequence of real numbers which is bounded above has a limit (see [8, Theorem 3.14]), $\alpha=\lim _{n \rightarrow \infty} a_{n}$ is a well-defined real number between 0 and 1 . Alice wins the game if $\alpha \in S$, and Bob wins if $\alpha \notin S$.

Countable and uncountable sets. An set $X$ is called countable if it is possible to list the elements of $X$ in a (possibly repeating) infinite sequence $x_{1}, x_{2}, x_{3}, \ldots$ Equivalently, $X$ is countable if there is a function from the set $\{1,2,3, \ldots\}$ of natural numbers to $X$ which is onto. For example, every finite set is countable, and the set of natural numbers is countable. A set which is not countable is called uncountable. Cantor proved using his famous diagonalization argument that the real interval $[0,1]$ is uncountable. We will give a different proof of this fact based on Alice and Bob's game.

Proposition 1. If $S$ is countable, then Bob has a winning strategy.

\footnotetext{
${ }^{1}$ Supported by NSF Grant DMS-0300784.
} 
Proof. Since $S$ is countable, one can enumerate the elements of $S$ as $s_{1}, s_{2}, s_{3}, \ldots$ Consider the following strategy for Bob. On move $n \geq 1$, he chooses $b_{n}=s_{n}$ if this is a legal move, and otherwise he randomly chooses any allowable number for $b_{n}$. Since $\alpha<b_{n}$ for all $n$, it follows that $\alpha \neq b_{n}$ for any $n \geq 1$, and thus $\alpha \notin S$. This means that Bob always wins with this strategy!

If $S=[0,1]$, then clearly Alice wins no matter what either player does. Therefore we deduce:

Corollary 1 . The interval $[0,1] \subset \mathbb{R}$ is uncountable.

This argument is in many ways much simpler than Cantor's original proof!

Perfect sets. We now prove a generalization of the fact that $[0,1]$ is uncountable. This will also follow from an analysis of our game, but the analysis is somewhat more complicated. Given a subset $X$ of $[0,1]$, we make the following definitions:

- A limit point of $X$ is a point $x \in[0,1]$ such that for every $\epsilon>0$, the open interval $(x-\epsilon, x+\epsilon)$ contains an element of $X$ other than $x$.

- $X$ is closed if every limit point of $X$ belongs to $X$.

- $X$ is perfect if it is non-empty ${ }^{2}$, closed, and if every element of $X$ is a limit point of $X$.

For example, the famous middle-third Cantor set is perfect (see [8, §2.44]). If $L(X)$ denotes the set of limit points of $X$, then a nonempty set $X$ is closed $\Leftrightarrow L(X) \subseteq X$, and is perfect $\Leftrightarrow L(X)=X$. It is a well-known fact that every perfect set is uncountable (see [8, Theorem 2.43]). Using our infinite game, we will give a different proof of this fact. We recall the following basic property of the interval $[0,1]$ :

\footnotetext{
${ }^{2}$ Some authors consider the empty set to be perfect.
} 
( $\star$ Every non-empty subset $X \subseteq[0,1]$ has an infimum (or greatest lower bound), meaning that there exists a real number $\gamma \in[0,1]$ such that $\gamma \leq x$ for every $x \in X$, and if $\gamma^{\prime} \in[0,1]$ is any real number with $\gamma^{\prime} \leq x$ for every $x \in X$, then $\gamma^{\prime} \leq \gamma$.

The infimum $\gamma$ of $X$ is denoted by $\inf (X)$.

Let's say that a point $x \in[0,1]$ is approachable from the right, denoted $x \in X^{+}$, if for every $\epsilon>0$, the open interval $(x, x+\epsilon)$ contains an element of $X$. We can define approachable from the left (written $x \in X^{-}$) similarly using the interval $(x-\epsilon, x)$. It is easy to see that $L(X)=X^{+} \cup X^{-}$, so that a non-empty set $X$ is perfect $\Leftrightarrow X=X^{+} \cup X^{-}$.

The following two lemmas tell us about approachability in perfect sets.

Lemma 1. If $S$ is perfect, then $\inf (S) \in S^{+}$.

Proof. The definition of the infimum in $(\star)$ implies that $\inf (S)$ cannot be approachable from the left, so, being a limit point of $S$, it must be approachable from the right.

Lemma 2. If $S$ is perfect and $a \in S^{+}$, then for any $\epsilon>0$, the open interval $(a, a+\epsilon)$ also contains an element of $S^{+}$.

Proof. Since $a \in S^{+}$, we can choose three points $x, y, z \in S$ with $a<x<$ $y<z<a+\epsilon$. Since $(x, z) \cap S$ contains $y$, the real number $\gamma=\inf ((x, z) \cap S)$ satisfies $x \leq \gamma \leq y$. If $\gamma=x$, then by $(\star)$ we have $\gamma \in S^{+}$. If $\gamma>x$, then $(\star)$ implies that $\gamma \in L(X)$ and $(x, \gamma) \cap S=\emptyset$, so that $\gamma \notin S^{-}$and therefore $\gamma \in S^{+}$.

From these lemmas, we deduce:

Proposition 2. If $S$ is perfect, then Alice has a winning strategy.

Proof. Alice's only constraint on her $n$th move is that $a_{n-1}<a_{n}<b_{n-1}$. By induction, it follows from Lemmas 1 and 2 that Alice can always choose $a_{n}$ to be an element of $S^{+} \subseteq S$. Since $S$ is closed, $\alpha=\lim a_{n} \in S$, so Alice wins!

From Propositions 1 and 2, we deduce:

Corollary 2. Every perfect set is uncountable. 
Further analysis of the game. We know from Proposition 1 that Bob has a winning strategy if $S$ is countable, and it follows from Proposition 2 that Alice has a winning strategy if $S$ contains a perfect set. (Alice just chooses all of her numbers from the perfect subset.) What can one say in general? A well-known result from set theory [1, §6.2, Exercise 5] says that every uncountable Borel set ${ }^{3}$ contains a perfect subset. Thus we have completely analyzed the game when $S$ is a Borel set: Alice wins if $S$ is uncountable, and Bob wins if $S$ is countable. However, there do exist non-Borel uncountable subsets of $[0,1]$ which do not contain a perfect subset [1, Theorem 6.3.7]. So we leave the reader with the following problem:

Problem: Do there exist uncountable subsets of $[0,1]$ for which: (a) Bob has a winning strategy; (b) Alice does not have a winning strategy; or (c) neither Alice nor Bob has a winning strategy?

Related games. Our infinite game is a slight variant of the one proposed by Jerrold Grossman and Barry Turett in [2] (see also 6] ). Propositions 1 and 2 above were motivated by parts (a) and (c), respectively, of their problem. The author originally posed Propositions 1 and 2 as challenge problems for the students in his Math 25 class at Harvard University in Fall 2000.

A related game (the "Choquet game") can be used to prove the Baire category theorem (see §8.C of [5] and [3]). In Choquet's game, played in a given metric space $X$, Pierre moves first by choosing a non-empty open set $U_{1} \subseteq X$. Then Paul moves by choosing a non-empty open set $V_{1} \subseteq U_{1}$. Pierre then chooses a non-empty open set $U_{2} \subseteq V_{1}$, etc., yielding two decreasing sequences $U_{n}$ and $V_{n}$ of non-empty open sets with $U_{n} \supseteq V_{n} \supseteq U_{n+1}$ for all $n$, and $\cap U_{n}=\cap V_{n}$. Pierre wins if $\cap U_{n}=\emptyset$, and Paul wins if $\cap U_{n} \neq \emptyset$. One can show that if $X$ is complete, then Paul has a winning strategy, and if $X$ contains a non-empty open set $O$ which is a countable union of closed sets

\footnotetext{
${ }^{3} \mathrm{~A}$ Borel set is, roughly speaking, any subset of $[0,1]$ that can be constructed by taking countably many unions, intersections, and complements of open intervals; see [․ §11.11] for a formal definition.
} 
having empty interior, then Pierre has a winning strategy. As a consequence, one obtains the Baire category theorem: If $X$ is a complete metric space, then no open subset of $X$ can be a countable union of closed sets having empty interior.

Another related game is the Banach-Mazur game (see $\S 6$ of [7] and $\S 8 . \mathrm{H}$ of [5]). A subset $S$ of the unit interval [0,1] is fixed, and then Anna and Bartek alternate play. First Anna chooses a closed interval $I_{1} \subseteq[0,1]$, and then Bartek chooses a closed interval $I_{2} \subseteq I_{1}$. Next, Anna chooses a closed interval $I_{3} \subseteq I_{2}$, and so on. Together the players' moves determine a nested sequence $I_{n}$ of closed intervals. Anna wins if $\cap I_{n}$ has at least one point in common with $S$, otherwise Bartek wins. It can be shown that Bartek has a winning strategy if and only if $S$ is meagre (see Theorem 6.1 of [7]). (A subset of $X$ is called nowhere dense if the interior of its closure is empty, and is called meagre, or of the first category, if it is a countable union of nowhere dense sets.) It can also be shown, using the axiom of choice, that there exist sets $S$ for which the Banach-Mazur game is undetermined (neither player has a winning strategy).

For a more thorough discussion of these and many other topological games, we refer the reader to the survey article 9], which contains an extensive bibliography. Many of the games discussed in [9] are not yet completely understood.

Games like the ones we have been discussing play a prominent role in the modern field of descriptive set theory, most notably in connection with the axiom of determinacy (AD). (See Chapter 6 of [4] for a more detailed discussion.) Let $X$ be a given subset of the space $\omega^{\omega}$ of infinite sequences of natural numbers, and consider the following game between Alice and Bob. Alice begins by playing a natural number, then Bob plays another (possibly the same) natural number, then Alice again plays a natural number, and so on. The resulting sequence of moves determines an element $x \in \omega^{\omega}$. Alice wins if $x \in X$, and Bob wins otherwise. The axiom of determinacy states that this game is determined (i.e., one of the players has a winning strategy) 
for every choice of $X$.

A simple construction shows that the axiom of determinacy is inconsistent with the axiom of choice. On the other hand, with Zermelo-Fraenkel set theory plus the axiom of determinacy $(\mathrm{ZF}+\mathrm{AD})$, one can prove many nontrivial theorems about the real numbers, including: (i) every subset of $\mathbb{R}$ is Lebesgue measurable; and (ii) every uncountable subset of $\mathbb{R}$ contains a perfect subset. Although $\mathrm{ZF}+\mathrm{AD}$ is not considered a "realistic" alternative to ZFC (Zermelo-Fraenkel + axiom of choice), it has stimulated a lot of mathematical research, and certain variants of $\mathrm{AD}$ are taken rather seriously. For example, the axiom of projective determinacy is intimately connected with the continuum hypothesis and the existence of large cardinals (see [10] for details).

\section{References}

[1] K. Ciesielski, Set Theory for the Working Mathematician, London Mathematical Society Student Texts 39, Cambridge University Press, 1997.

[2] J. W. Grossman and B. Turett, Problem \#1542, Mathematics Magazine 71, February 1998.

[3] F. Hirsch and G. Lacombe, Elements of Functional Analysis, Graduate Texts in Mathematics 192, Springer-Verlag, 1999.

[4] A. Kanamori, The Higher Infinite (2nd ed.), Springer-Verlag, 2003.

[5] A. Kechris, Classical Descriptive Set Theory, Springer-Verlag, 1995.

[6] W. A. Newcomb, Solution to Problem \#1542, Mathematics Magazine 72, February 1999.

[7] J. Oxtoby, Measure and Category (2nd ed.), Springer-Verlag, 1980. 
[8] W. Rudin, Principles of Mathematical Analysis (3rd ed.), McGraw-Hill, 1976.

[9] R. Telgársky, Topological games: On the 50th anniversary of the Banach-Mazur game, Rocky Mountain J. Math. 17 (1987), 227-276.

[10] H. Woodin, The Continuum Hypothesis, Part I, Notices of the AMS 48, no. 6 (2001), 567-576. 